\title{
FAULT-TOLERANT TOPOLOGY CONTROL FOR IOT NETWORKS USING VARIABLE K-CONNECTIVITY
}

\author{
Mitsumasa Ota ${ }^{1}$, Ryuichi Takahashi ${ }^{2}$ and Yoshiaki Fukazawa ${ }^{1}$ \\ ${ }^{1}$ Waseda University, 3-4-1 Okubo, Shinjuku-ku, Tokyo 169-8555, Japan \\ ${ }^{2}$ Ibaraki University, 4-12-1 Nakanarusawa-cho. Hitachi-shi, Ibaraki 316-8511, Japan
}

\begin{abstract}
Various devices communicate in the Internet of Things (IoT). However, not all devices have direct communication capabilities due to device constraints such as size and cost. To overcome such limitations, multi-hop communications with gateways are used. Advantages of using gateways include downsized devices and reduced operation costs. When each node communicates with maximum power in multi-hop communications, power consumption increases unnecessarily. Therefore, the communication power of each node is adjusted using topology control. Typically, topology control reduces communication power. As the number of edges between nodes decreases, the more serious the impact of node failure. In topology control, this problem is addressed by considering k-vertex connectivity. For k-vertex connectivity, the larger the value of $\mathrm{k}$, the higher the power consumption. Existing fault-tolerant topology control assumes that similar data is transmitted and the environment has a uniform $\mathrm{k}$ value. Since various types of data are communicated in an IoT environment, the connectivity requirements vary. Herein a method is proposed to realize a variable $\mathrm{k}$ value to eliminate excessive connectivity and reduce power consumption. The proposed method uses the genetic algorithm (GA). To obtain a correct solution by GA, we propose an encoding scheme and define a fitness function. Simulation experiments evaluated the proposed method and compared the results with existing fault-tolerant topology control. The proposed method can construct a more power efficient topology than the existing topology control method.
\end{abstract}

\section{KEYWORDS}

Internet of Things(IoT), Topology Control, k-Connectivity, Genetic Algorithm (GA)

\section{INTRODUCTION}

In an Internet of Things (IoT) environment, resources such as radiofrequency identification (RFIDs), sensors, and actuators installed in various "things" communicate with and control each other (Atzori et al., 2010). IoT is rapidly spreading due to the miniaturization of power devices and the development of new communication technologies (Rawat et al., 2016). Currently, IoT environments range from small scale IoT (called nano IoT) to global scale IoT (Yuichi et al., 2014). It is predicted that 50 billion devices will be connected to the Internet in 2020 (Evans, 2011).

Many devices are driven by batteries. Although numerous resources exchange data, not all can interconnect directly with geographically remote resources due to limited battery capacities. In such a case, gateways are used. Figure 1(a) shows a configuration without a gateway, where devices must be capable of long-distance communication to transmit data directly to other devices. Figure 1(b) shows a configuration with wireless multi-hop communications using gateways. A gateway functions as a relay point for communication, allowing devices to communicate. Gateways can reduce the required communication distance for each device. Other advantages include device miniaturization and cost and power consumption reductions. 


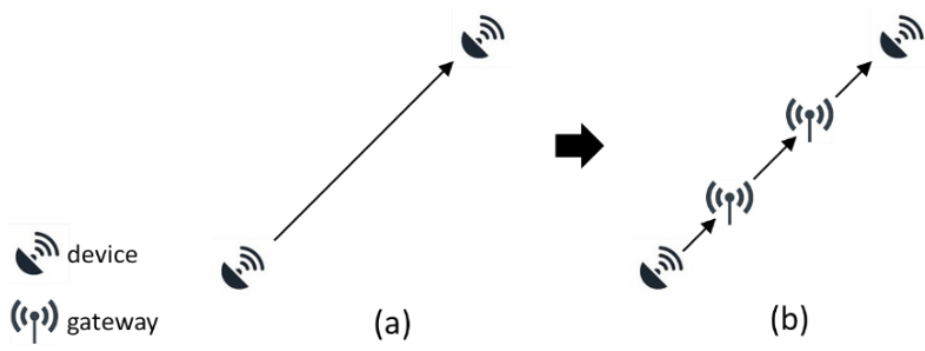

Figure 1. Network configuration (a) without gateways and (b) with gateways

In wireless multi-hop communications, multiple paths are prepared between two points in a network to prevent disconnection due to node failure. Topology control is used to derive redundant paths with regard to power efficiency. It is a technology to minimize power consumption in multi-hop communications as the transmission power of each node is adjusted to minimize the power consumption in the environment while maintaining the required connectivity of the network.

In topology control, $k$-connectivity is used to consider fault tolerance. When there are $k$ independent paths (paths that do not share nodes other than the start and end points) from node $u$ to $v$, then $u$ and $v$ are $k$-connected. Since the connectivity between $k$-connected nodes is maintained even if a number of nodes up to $k$ are removed, the communication between $u$ and $v$ is tolerant to at least $k$ - 1 node failures. If two arbitrary nodes in the graph are $k$-connected, the graph is called a $k$-connected graph.

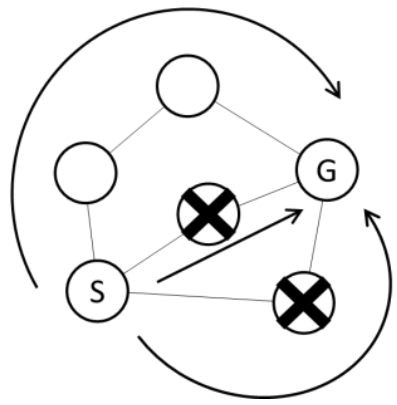

Figure 2. 3-connected nodes

Figure 2 shows that nodes $\mathrm{S}$ and $\mathrm{G}$ are connected by three independent paths (3-connectivity). The connection between $S$ and $G$ is maintained when 3-1=2 nodes are removed. For example, one path is maintained even if the nodes with the cross in Figure 2 are removed.

In a k-connected graph, the degree of each node (the number of edges joined to the node) must be greater than or equal to $\mathrm{k}$. Because each node is connected to many nodes, the power consumption increases as $\mathrm{k}$ increases. Therefore, power consumption and fault tolerance have a trade-off relationship.

The existing topology control method proposed in the field of wireless sensor networks (WSNs) and ad hoc networks uses a fixed $\mathrm{k}$ value in the environment. In these fields, communication of homogeneous data is assumed. However, diverse data are exchanged in IoT applications with varying levels of importance. Consequently, not all communication paths have the same fault tolerance in the IoT.

Consider a factory system controlled by IoT as an example. This system consists of production machines, fault detection sensors, and sensors for energy usage and production volume. These devices are interconnected via wireless gateways. This system allows the production volume to be monitored, the energy consumption to be analyzed, and the production line to be halted immediately if a machine fault occurs. In this example, data such as detection of a machine fault is highly important and real-time transmission is required. Therefore, a high fault tolerance is required to prevent a delay due to gateway failure. However, not all data in the system needs to be real-time. A delay due to retransmission is permitted when a high degree of real-time performance is not required such as monitoring the energy consumption and production volume. Maintaining a high fault tolerance for all information can lead to inefficient power consumption. 
As in the example above, in IoT, a high fault tolerance is not necessary between all devices. However, maintaining a high fault tolerance may increase power consumption. Herein we propose a method to reduce the power consumption while maintaining the connectivity by setting the value of $\mathrm{k}$ for each set of devices.

The remainder of this paper is organized as follows. Chapter 2 describes related work. Chapter 3 details the problem, while Chapter 4 proposes a method to solve the problem. Chapter 5 explain the experiments. Finally, Chapter 6 provides the conclusions.

\section{RELATED WORK}

This section introduces related research. Some studies have investigated networks using gateways. Kim et al. (2015) investigated a method to solve the resource allocation problem to a gateway. Their method converts the resource allocation problem into a minimum spanning tree problem, which is solved by a genetic algorithm (GA). Although their method calculates the tree topology to minimize data transmission cost, it does not assume the occurrence of gateway failure. With regard to fault tolerant topology, their method differs from our method.

Karthikeya et al. (2003) proposed an optimization method for gateway placement. Their approach minimizes the installation cost of gateways by optimizing gateway placement. The optimum gateway placement is calculated only where devices are deployed. However, we propose a method to optimize the topology in an environment where devices and gateways are already deployed.

Ismail et al. (2012) examined a resource allocation method assuming a mobile network in a device called a mobile terminal. In their research, resource allocation means to determine the access point to which each mobile terminal is connected. Since their method is a distributed algorithm to find an optimal solution for each partitioned network, one advantage is that a central resource manager is unnecessary. In their method, the mobile terminal corresponds to the IoT device, and the network base station and access point operate like a gateway in an IoT environment. The problem handled by their method is similar to the problem dealt with in our research as both assume that device communications use relay points. However, the other assumptions differ. In their method, each mobile terminal can connect to multiple networks simultaneously, whereas in ours, each IoT device is allocated to one gateway chosen from the candidates. Additionally, they do not assume node failure.

Other studies have examined tolerant topology control using k-connectivity. The problem of finding the minimum cost k-connected graph is NP-hard, and many approximation algorithms have been proposed (Hajiaghayi et al., 2003) (Khuller, 1997).

$\mathrm{Li}$ and Hou (2004) proposed FGSS and FLSS, which are fault tolerant topology control algorithms with k-connected graphs. These algorithms extend the Kruskal algorithm (Kruskal, 1956), which is a basic algorithm to find the minimum spanning tree (1-connected graph with the minimum sum of path costs). It constantly monitors the network and judges whether the network maintains k-connectivity.

$\mathrm{Li}$ et al. (2005) proposed the CBTC algorithm to maintain k-connectivity in a network. In the topology obtained by this algorithm, k-connectivity is maintained between two arbitrary nodes. However, k-connectivity is unnecessary between arbitrary two nodes, and it is sufficient to maintain k-connections only between nodes that communicate. Both of these algorithms can determine a k-connection graph. In other words, they do not consider which nodes are communicating.

In contrast, Xiao and Zhou (2013) proposed an FTCGA algorithm to reduce energy consumption by guaranteeing k-connectivity only between sensor nodes and sink nodes in a WSN. This method calculates the topology with a reduction in redundant links while maintaining k-connectivity only between communicating nodes using a GA.

In fault tolerant topology control, reducing the power consumption by eliminating unnecessary links is emphasized. Our method aims to reduce links while maintaining the required connectivity by setting the $\mathrm{k}$ value for each pair of devices that communicate instead setting a uniform $\mathrm{k}$ value for the environment.

\section{PROBLEM DEFINITION}

This chapter defines the problem this study aims to address. The problem environment is constructed of two kinds of devices: an IoT gateway and a gateway. Devices communicate in the deployed environment. 
Gateways function as relay points of communication between devices. The gateway $g_{i}$ has its own position $\left(x_{i}, y_{i}\right)$ and maximum transmission radius $r_{\max }\left(g_{i}\right)$, which is the maximum distance that data can be transmitted and this value is static. The actual transmission radius $r\left(g_{i}\right)$ can be changed in the range $0 \leq r\left(g_{i}\right) \leq r_{\max }\left(g_{i}\right)$. As shown in Figure 3, the gateway can transmit data to other gateways that are within the transmission radius. However, it cannot transmit data directly to gateways that are beyond its transmission radius. In other words, there is an edge from gateway $g_{i}$ to gateway $g_{j}$ if $r\left(g_{i}\right) \geq \operatorname{dis}(i, j)$, where $\operatorname{dis}(i, j)$ is the distance between $g_{i}$ and $g_{j}$.

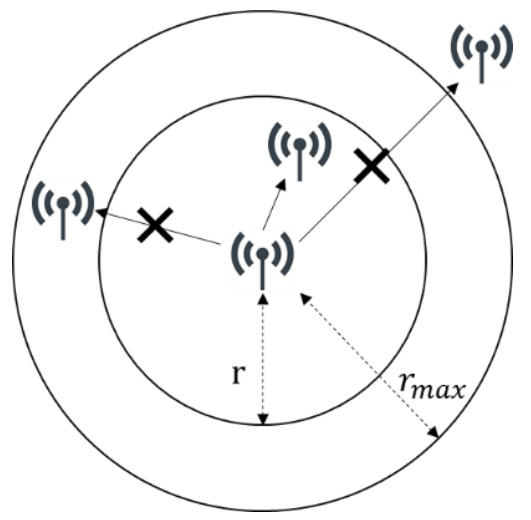

Figure 3. Gateway can transmit data directly to the gateway within transmission radius

Devices, which transmit and receive data, are the end points of a communication path. The device $d_{i}$ is allocated to one gateway al $\left(d_{i}\right)$ to communicate via gateways. Every device has a list of allocatable gateways, which must have at least one element.

From these condition, the problem environment is represented by the directed graph $G$. $G$ is as follows.

$$
\begin{aligned}
& G=(V, E) \\
& V=V_{d} \cup V_{g} \\
& V_{d}=\left\{d_{n} \mid n=\text { device } I D\right\} \\
& V_{g}=\left\{g_{n} \mid n=\text { gateway } I D\right\} \\
& E=E_{d} \cup E_{g} \\
& E_{d}=\left\{(d, g) \mid d \in V_{d}, g \in V_{g}, g=a l(d)\right\} \\
& E_{g}=\left\{\left(g_{i}, g_{j}\right) \mid g_{i} \in V_{g}, g_{j} \in V_{g}, r\left(g_{i}\right) \geq \operatorname{dis}\left(g_{i}, g_{j}\right)\right\}
\end{aligned}
$$

The directed graph $G$ is constructed by a set of nodes $V$ and a set of edges $E$. $V$ contains a set of device nodes $V_{d}$ and set of gateway nodes $V_{g} . E$ consists of two edge sets: $E_{d}$ and $E_{g}$. Each edge $(d, g)$ included in $E_{d}$ represents the allocation of device $d$ to gateway $g$. Each edge $\left(g_{i}, g_{j}\right)$ included in $E_{g}$ means that gateway $g_{i}$ can transmit data directly to gateway $g_{j}$.

For such an environment, an input is given by $T=\left\{t=\left(d_{i}, d_{j}, k\right) \mid d_{i}, d_{j} \in V_{d}, k>0\right\} . t \in \mathrm{T}$ represent a pair of devices that communicate and their required connectivity. $t=\left(d_{i}, d_{j}, k\right)$ means that $k$-connectivity is required between device $d_{i}$ and $d_{j}$. Because the $k$ value can be set for each pair of devices, our method differs from existing fault tolerant topology control using a fixed $k$ value.

The output is the allocation $a l(d)$ of all devices and the transmission radius $r(g)$ of all gateways. Since the transmission power in wireless communication is the square of the transmission distance, the power consumption in gateway $g_{i}$ is represented by e $\left(g_{i}\right)=\mathrm{r}\left(g_{i}\right)^{2}$. In this paper, we aim to find $a l(d)$ and $r(g)$ to minimize the sum of the power consumption in the environment while maintaining the required connectivity.

\section{PROPOSED METHOD}

We propose a method to obtain solutions to the problems defined in Chapter 3. Since the problem of finding the minimum cost $k$-connected graph is proven to be NP-hard, our method uses a GA to determine transmission radius of each gateway and allocation. GA is a heuristic algorithm for approximate solutions by evolving a set of candidates encoded by a process that imitates evolution (Beasley, 1993). In this paper, we define the 
encoding scheme and the fitness function to evaluate the candidate that minimizes the power consumption while maintaining the required connectivity. In addition, generation of the next candidate is done by a one-point crossover and mutation. The encoding scheme and fitness function are described below.

\subsection{Encoding Scheme}

In a GA, candidates are represented by a sequence. This is called the code of candidates. In this subsection, we describe the encoding scheme.

Each candidate is represented by following code $C$

$$
\begin{gathered}
C=\operatorname{Gene}[D+G] \\
\text { Gene }[i]= \begin{cases}\operatorname{al}\left(d_{i}\right) & (i<D) \\
r\left(g_{i-D}\right) & (i \geq D)\end{cases}
\end{gathered}
$$

where $D$ is the number of devices and $G$ is the number of gateways. $a l\left(d_{i}\right)$ represents the ID of the gateway in which the device $d_{i}$ is allocated and $r\left(g_{i}\right)$ represents the transmission radius of gateway $g_{i}$. That is, the code of each candidate is an integer sequence where the length is the sum of the number of gateways and devices. The code is divided into two. The first (former) part shows the gateway to which each device is allocated, and the second (latter) part shows the transmission radius of each gateway.

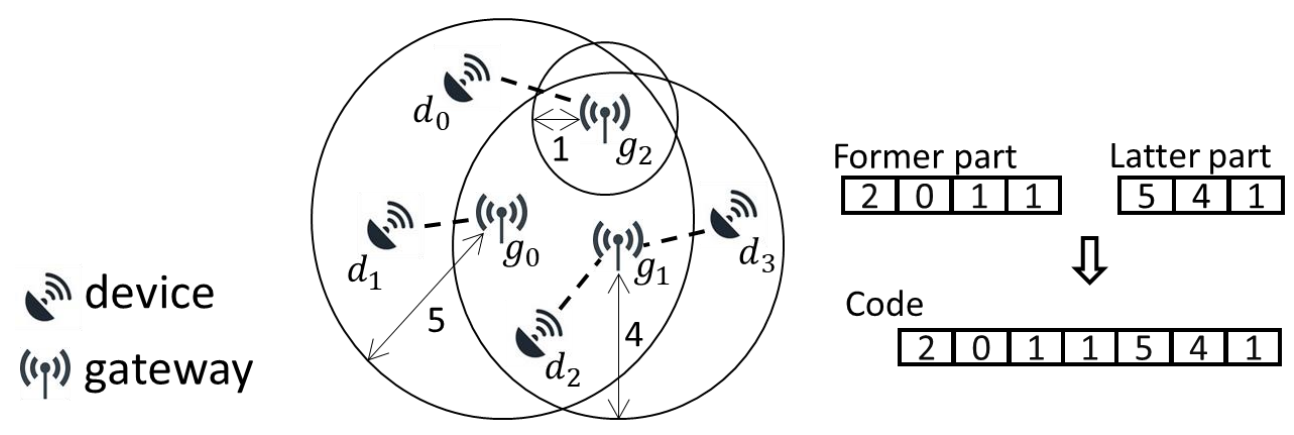

Figure 4. Example of encoding

Figure 4 shows an example of encoding by this scheme, where edges between gateways are omitted for simplicity. The device $d_{0}$ is allocated to the gateway $g_{2}$, so Gene[0] $=2$. Similarly, the former part is "2011" for the other devices. Because $r\left(g_{0}\right)=5$, Gene $[4]=r\left(g_{4-4}\right)=r\left(g_{0}\right)=5$. Considering the other gateways in the same manner, the latter is "541". Thus, the code representing the state in Figure 4 is "2011541."

The encoding considers two properties: locality and heritability. Locality is the influence of each element of the code on the structure of the solution. Encoding with a low locality means a small change in the code may greatly change the structure of the solution. Heritability is the how much a child candidate inherits the characteristics of a parent by crossover. A low heritability means that a child will not inherit the parent's characteristics, which prevents the child from obtaining a better solution.

Regarding the locality in our encoding scheme, each element of the former part represents the allocation of one device, and each element of the latter part represents the transmission radius of one gateway. If an element of the former is changed, only one device allocation is changed. The topology of the other part is not affected. Because the transmission radius is related only to the existence of the edge between one gateway and its adjacent gateways, a change in the topology due to a change in the transmission radius is local. Therefore, the locality of our encoding scheme is high.

In this encoding scheme, a child topology inherits the edge in the parent topology corresponding to the code inherited from the parent. Consequently, it also has a high heritability.

\subsection{Fitness Function}

This subsection describes the fitness function. Fitness represents how good each candidate in the GA is. Candidates with a higher fitness calculated by the fitness function are obtained as solution. In our method, the 
fitness function is defined to evaluate whether each candidate minimizes the power consumption while maintaining the required connectivity.

The fitness function is as follows.

$$
\begin{aligned}
& \text { fitness }=\sum_{g_{i} \in \vec{\forall}_{g}} e\left(g_{i}\right)+\sum_{t \in T} p * s(t) \\
& \mathrm{e}\left(g_{i}\right)=\mathrm{r}\left(g_{i}\right)^{2} \\
& p=\sum_{g_{i} \in \vec{v}_{g}}\left\{r_{\max }\left(g_{i}\right)\right\}^{2} \\
& s(t)=\max \left(t . k-n_{\text {path }}\left(t . d_{i}, t . d_{j}\right), 0\right)
\end{aligned}
$$

The first term represents the total power consumption. Candidates with low values are evaluated highly.

The second term represents a penalty when the required connectivity is not satisfied. Here, $s(t)$ represents the number of missing paths for $\mathrm{t}=\left(d_{i}, d_{j}, \mathrm{k}\right) . n_{\text {path }}\left(d_{i}, d_{j}\right)$ represents the number of independent paths existing between the gateways to which the devices $d_{i}, d_{j}$ are allocated. When the value does not satisfy the required connectivity, $s(t)$ returns the number of missing paths. $p$ is a penalty when the required connectivity is not satisfied. By setting the penalty proportional to the number of missing paths, it is possible to evaluate a candidate that does not satisfy the required connectivity lower than others. In our method, because an approximate solution is obtained by GA, the obtained solution does not necessarily satisfy the required connectivity. However, since the value of $p$ is equal to the total power consumption when each gateway communicates at its maximum transmission radius, candidates that do not satisfy the required connectivity are always evaluated lower than candidates that do satisfy it.

\section{EVALUATION}

We conducted simulation experiments to evaluate whether the proposed method reduces the power consumption. In the experiment, topologies are obtained for randomly generated inputs. The required connectivity $k$ is $1 \leq k \leq 3$.

Experiment 1 shows the case where 10 environments consisting of 20 gateways and 50 devices are randomly generated. The power consumption for three methods are compared: the proposed method, the FGSS algorithm ( $\mathrm{Li}$ and $\mathrm{Hou}, 2004)$, and without topology control (the case where each gateway communicates at its maximum transmission radius). In Experiment 2, the execution time as the number of gateways is changed for 100 devices is compared between FGSS and the proposed method.

The experiments were conducted on a PC running in the Windows 10 (32 bit) environment equipped with Intel ${ }^{\circledR}$ Core $^{\mathrm{TM}}$ i5 CPU M560 2.67GHz and $4.00 \mathrm{~GB}$ memory. In the GA application, the number of candidates was $N=50$, the number of selected candidates was $N_{\text {select }}=10$, the maximum number of generations was gen $=50$, and the mutation probability was $P_{M}=0.10$.

\subsection{Experiment 1: Power Consumption}

In this subsection, we evaluate whether the proposed method can reduce power consumption. The proposed method is compared with the FGSS algorithm (existing fault tolerant topology control) as well as the case without topology control.

Figure 5 shows the total of power consumption in the topology generated with 20 gateways and 50 devices.

The proposed method can reduce the power consumption. Compared with configurations without topology control, FGSS reduces the power consumption by about $15 \%$, whereas the proposed method reduces it by about $30 \%$. In other words, it is possible to reduce the power consumption by topology control. The proposed method provides a topology with a higher power efficiency. In FGSS, 3-connectivity is guaranteed between all nodes. Hence, even devices that do not communicate with each other have three connections. As a result, the power consumption in FGSS increases as the number of links increases. On the other hand, the proposed method reduces the links for parts that do not require a high connectivity, which improves the power efficiency. 


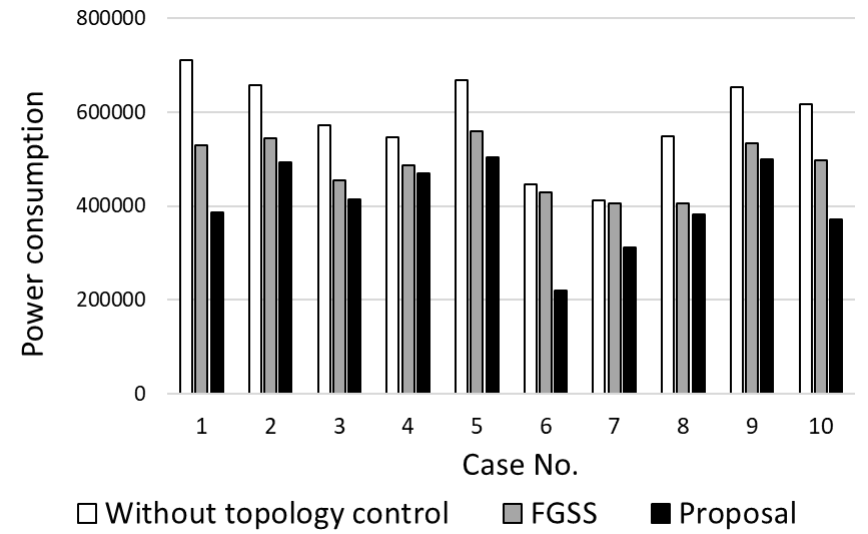

Figure 5. Power consumption

In some cases, FGSS does not significantly reduce the power consumption (Figures 5, No. 6 and No. 7). This may be because the topology without topology control is similar to the $k$-connected graph. Thus, the $k$-connectivity cannot be maintained if the transmission power is reduced. Moreover, the power consumption of the proposed method is closer to that of FGSS in No. 3 and No. 8. It is thought that many of the required connectivities have $k=3$. Hence, setting $k$ individually does not have a significant impact on removing unnecessary connections.

The results show that the proposed method can provide a topology with a higher power efficiency. The effect is considered to be greater in the case where the required connectivity varies.

\subsection{Experiment 2: Execution Time}

In this subsection, we evaluate the execution time to calculate the topology in the proposed method. The results are compared with FGSS.

Figure 6 shows the execution time for 100 devices. The execution time of the proposed method is longer than that of FGSS when the gateway is small. Since the execution time of FGSS is proportional to the number of edges in the graph ( $\mathrm{Li}$ and Hou, 2004), as the number of gateways increases, the execution time increases exponentially. On the other hand, in the proposed method, the increase in execution time due to the increase in the number of gateways is small, suggesting that the proposed method is useful in the IoT, where more devices need to be considered.

he execution time of the proposed method is about 10 seconds when there are 40 gateways. Since the $k$ value of the required connectivity can be set from the occurrence probability of node failure and the calculation time, the proposed method can calculate the topology with a practical execution time.

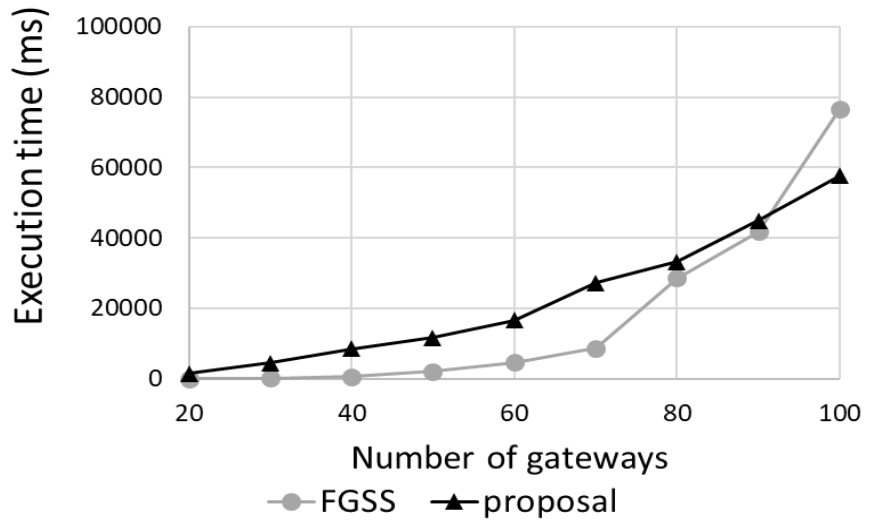

Figure 6. Execution time 


\section{CONCLUSION}

Multi-hop communications using gateways are implemented because individual device constraints prevent all devices from communicating directly with each other. The existing fault tolerant topology control uses $k$-connectivity, but it assumes communications occur between homogeneous devices and a fixed $k$ value. However, the required connectivity varies in the IoT since communication occurs between heterogeneous devices.

Herein we propose a method that employs a GA and varies the $k$ value for each pair. Simulation experiments show that our method can reduce power consumption. Although our method can obtain an energy efficient topology, the calculation time is similar to the existing method. For a dynamic IoT, the time efficiency of the algorithm should be improved.

In the future, the practicality of the method through experiments in an actual IoT environment should be evaluated as this study was a simulation in a randomly generated environment.

\section{REFERENCES}

Beasley, D. et al, 1993. An overview of genetic algorithms: Part 1, fundamentals. University computing, Vol. 15, No. 2, pp. 56-69.

Evans, D., 2011. The Internet of Things: How the next evolution the Internet is changing everything. CISCO white paper.

Hajiaghayi, M. et al, 2003. Power optimization in fault-tolerant topology control algorithms for wireless multi-hop networks. Proceedings of the 9th annual international conference on Mobile computing and networking, San Diego, California, USA, pp. 300-312.

Ismail, M. and Zhuang, W., 2012. A distributed multi-service resource allocation algorithm in heterogeneous wireless access medium. IEEE Journal on Selected Areas in Communications. Vol. 30, No. 2, pp. 425-432.

Karthikeya, S. A. et al, 2016. Leveraging solution-specific gateways for cost-effective and fault-tolerant IoT networking. IEEE Wireless Communications and Networking Conference, Doha, Qatar, pp. 1-6.

Khuller, S., 1998. Approximation algorithms for finding highly connected subgraphs. Approximation algorithms for NP-hard problems, pp. 236-265.

Kim, M. and Ko, I. Y., 2015. An efficient resource allocation approach based on a genetic algorithm for composite services in IoT environments. IEEE International Conference on Web service, New York, the US, pp. 543-550.

Kruskal, J. B., 1956. On the shortest spanning subtree of a graph and the traveling salesman problem. Proceedings of the American Mathematical society, Vol. 7, No. 1, 48-50.

Li, L. et al, 2005. A cone-based distributed topology-control algorithm for wireless multi-hop networks. IEEE/ACM Transactions on networking, Vol. 13, No.1, pp. 147-159.

Li, N., and Hou, J. C., 2004. FLSS: a fault-tolerant topology control algorithm for wireless networks. In Proceedings of the 10th annual international conference on Mobile computing and networking, Philadelphia, PA, USA, pp. 275-286.

Rawat, P. et al, 2016. Cognitive radio for M2M and Internet of Things: A survey. Computer communications, Vol. 94, pp. 1-29

Xiao, X., and Zhou, Z., 2013. Study on WSN's Fault-Tolerant Topology Control Based on GA. International Conference on in Computer Sciences and Applications, Delhi, India, pp. 146-149.

Yuichi, K. et al, 2014. Internet of Things (IoT): present state and future prospects. IEEE Transactions on Informartion and Systems, Vol. 97-D, pp. 2568-2575. 